\title{
Sarcopenia as a leading risk factor for erosive esophagitis
}

\author{
Tae Jun Kim¹, Jeung Hui Pyo², Hyuk Lee ${ }^{1}$, Seungho Ryu ${ }^{3,4,5}$, Sun-Young Baek ${ }^{6}$, \\ Kyunga Kim${ }^{6}$, Yang Won Min ${ }^{1}$, Byung-Hoon Min ${ }^{1}$, Jun Haeng Lee ${ }^{1}$, Hee Jung Son ${ }^{1,2}$, \\ Poong-Lyul Rhee ${ }^{1}$ and Jae J Kim ${ }^{1}$ \\ ${ }^{1}$ Department of Medicine, Samsung Medical Center, Sungkyunkwan University School of Medicine, Seoul, Korea \\ ${ }^{2}$ Center for Health Promotion, Samsung Medical Center, Sungkyunkwan University School of Medicine, Seoul, Korea \\ ${ }^{3}$ Center for Cohort Studies, Total Healthcare Center, Kangbuk Samsung Hospital, Sungkyunkwan University School of \\ Medicine, Seoul, Republic of Korea \\ ${ }^{4}$ Department of Occupational and Environmental Medicine, Kangbuk Samsung Hospital, Sungkyunkwan University School \\ of Medicine, Seoul, Republic of Korea \\ ${ }^{5}$ Department of Health Sciences and Technology, Samsung Advanced Institute for Health Sciences and Technology, \\ Sungkyunkwan University, Seoul, Republic of Korea \\ ${ }^{6}$ Statistics and Data Center, Samsung Medical Center, Sungkyunkwan University School of Medicine, Seoul, Korea \\ Correspondence to: Hyuk Lee, email: leehyuk@skku.edu
}

Keywords: sarcopenia; obesity; erosive esophagitis; reflux esophagitis; risk factors

Received: July 04, $2017 \quad$ Accepted: November 26, $2017 \quad$ Published: January 10, 2018

Copyright: Kim et al. This is an open-access article distributed under the terms of the Creative Commons Attribution License 3.0 (CC BY 3.0), which permits unrestricted use, distribution, and reproduction in any medium, provided the original author and source are credited.

\section{ABSTRACT}

Backgrounds: Obesity is an established risk factor for erosive esophagitis. Yet, the associations of sarcopenia and obesity with erosive esophagitis remain unclear. We studied the associations of obesity, sarcopenia, and sarcopenic obesity with the risk of erosive esophagitis in a large number of asymptomatic men and women.

Materials and Methods: We conducted a cross-sectional study of 32,762 asymptomatic adults undergoing routine health check-ups including screening endoscopy, between August 2006 and December 2011. Sarcopenia was defined as an appendicular skeletal muscle mass (ASM) / body weight value beyond two standard deviations below the mean for healthy young adults. The ASM was estimated using bioelectrical impedance analysis.

Results: Participants were categorized into four groups according to their obesity and sarcopenic status: normal, obese, sarcopenic, and sarcopenic obese. In a multivariable model adjusted for age, sex, smoking status, alcohol intake, regular exercise, and metabolic variables, the risk of erosive esophagitis was higher in obese [adjusted odds ratio(aOR), 1.38; 95\% confidence interval (CI), 1.26-1.52], sarcopenic (aOR, 2.20; 95\% CI, 1.48-3.29), and sarcopenic obese participants (aOR, 1.68; 95\% CI, 1.39-2.03) than in normal participants. Comparing sarcopenic and sarcopenic obese participants to obese participants, the ORs for erosive esophagitis were 1.59 (95\% CI, 1.06-2.38) and 1.22 (95\% CI, 1.02-1.47), respectively. In dose-response analyses, increasing sarcopenia severity showed a positive and graded relationship with overall, LA-B or higher grade, and LA-C erosive esophagitis.

Conclusions: Our findings suggest that sarcopenia, regardless of obesity status, is strongly and progressively associated with the risk of erosive esophagitis.

\section{INTRODUCTION}

Gastroesophageal reflux disease is a widespread gastrointestinal disorder that frequently occurs in primary care settings, imposing considerable burdens on global health and economics [1]. Disease prevalence is 18.1$27.8 \%$ in North America, 8.8-25.9\% in Europe, and 2.5$7.8 \%$ in East Asia, with rising rates worldwide [2]. Obesity is considered a significant contributing factor for a spectrum of reflux-related esophageal disorders ranging from 
erosive esophagitis to Barrett's esophagus to esophageal adenocarcinoma [3, 4]. Although the exact mechanisms have not been fully identified, several studies have demonstrated that the pattern of body fat distribution may be more important than general adiposity for increasing the risk of erosive esophagitis $[5,6]$. In addition to the increased intra-abdominal pressure caused by visceral adiposity, metabolically active visceral adipose tissue creates a proinflammatory and insulin-resistant condition [7-9]. Thus, the reflux-independent effect of adiposity on erosive esophagitis may contribute to the association, which cannot be solely explained by the mechanical effect of obesity.

Recent interest has focused on the age-related changes in body composition called sarcopenia. Sarcopenia is a pathological condition characterized by the progressive loss of muscle mass and increased amount of visceral fat, despite a relatively constant body weight [10]. Recent evidence shows that sarcopenia and sarcopenic obesity are associated with several cardiometabolic diseases, as well as with poor morbidity and mortality outcomes [11-17]. Reflux esophagitis (RE) and sarcopenia seem to have similar pathophysiologic backgrounds with regard to body composition and metabolic abnormalities. However, the effect of sarcopenia on erosive esophagitis has not been evaluated in a large population. Therefore, we examined the association between sarcopenia or sarcopenic obesity and the risk of erosive esophagitis. Furthermore, we evaluated whether sarcopenia or sarcopenic obesity is more likely to result in erosive esophagitis than is obesity in the absence of sarcopenia.

\section{RESULTS}

\section{Baseline characteristics of the study participants}

Among the 32,762 participants (mean age, 50.1 \pm 8.6 years), the prevalence of erosive esophagitis was $7.6 \%(n=2,493)$. According to the Los Angeles (LA) classification of erosive esophagitis, the prevalence of erosive esophagitis was $6.3 \%(n=2,063)$ of LA-A, $1.2 \%(n$ $=408)$ of LA-B, and $0.07 \%(n=22)$ of LA-C. There were no cases of LA grade D erosive esophagitis in this study of asymptomatic participants. The baseline characteristics of the participants are shown in Table 1 organized according to their obesity and sarcopenic status (normal, obese, sarcopenic, and sarcopenic obese groups), with significant differences observed among groups. The sarcopenic groups (sarcopenic obese and sarcopenic) were older, more likely to be male and not exercise regularly, had higher systolic blood pressures, had higher triglyceride, fasting glucose, and high sensitivity C-reactive protein (hsCRP) levels, and had lower ASM/weight, ASM/body mass index (BMI), and high density lipoprotein cholesterol (HDL-C) values; higher proportions of current smokers and modest alcohol consumers were also present in these groups than in the non-sarcopenic groups (normal and obese). Participants in the obese groups (obese and sarcopenic obesity) had higher proportions of current smokers, modest alcohol consumers, and had higher BMI and waist circumference values than did the non-obese groups (normal and sarcopenic).

\section{Risk of erosive esophagitis according to obesity and sarcopenia status}

Among the 32,762 participants, the prevalences of erosive esophagitis in the four groups was $6.0 \%$ (normal), $11.1 \%$ (obese), $15.2 \%$ (sarcopenic), and 14.8\% (sarcopenic obese) (Figure 1). Participants in the sarcopenic, and sarcopenic obese groups had higher prevalence of each grade of erosive esophagitis as well as overall erosive esophagitis $(P<0.001)$. Table 2 shows the risks of erosive esophagitis, based on obesity and sarcopenia status. Compared with the normal group, the obese, sarcopenic, and sarcopenic obese groups were more highly associated with erosive esophagitis. These associations were attenuated, but persisted, after adjusting for potential confounding factors and metabolic risk factors. In addition, the sarcopenic (nonobese) and sarcopenic obese groups were at higher risk of erosive esophagitis than were participants in the obese, nonsarcopenic group (Table 3). These associations persisted after adjusting for potential confounders and metabolic parameters. In the multivariable analysis, male sex, current smoking, modest alcohol intake ( $>10 \mathrm{~g} /$ day), and metabolic risk factors (e.g., systolic blood pressure, fasting blood glucose level, and triglyceride level) were independent risk factors for erosive esophagitis (data not shown).

\section{Risk of erosive esophagitis by grading according to sarcopenia status}

Table 4 shows the dose-response relationship between sarcopenia and erosive esophagitis. In models adjusted for age, sex, waist circumference, smoking status, alcohol intake, and exercise, adjusted ORs (95\% CIs) for overall erosive esophagitis comparing class I sarcopenia and class II sarcopenia with normal participants were 1.19 (1.08-1.32) and $1.51(1.26-1.81)$, respectively. In the dose-response analyses, increasing sarcopenia severity also showed a positive and graded dose-response relationship with erosive esophagitis LA-B or higher grade and LA-C. Further, the adjusted OR associated with a $1 \%$ decrease when ASM/weight was introduced as a continuous variable in regression models was 1.04 (1.011.06) for overall erosive esophagitis. The adjusted ORs for LA-B or higher grade and LA-C were 1.07 (1.01-1.13) and $1.30(1.10-1.55)$, respectively.

\section{Subgroup analyses of erosive esophagitis risk by obesity and sarcopenia status}

To evaluate the consistency of the effect of obesity and sarcopenia status on erosive esophagitis, we 
Table 1: Baseline characteristics of study participants according to obesity and sarcopenia status

\begin{tabular}{|c|c|c|c|c|c|}
\hline & $\begin{array}{c}\text { Normal } \\
(n=22,810)\end{array}$ & Obese $(n=8,533)$ & $\begin{array}{l}\text { Sarcopenic } \\
(n=311)\end{array}$ & $\begin{array}{c}\text { Sarcopenic } \\
\text { obese }(n=1,108)\end{array}$ & $P$ value \\
\hline Age (years) & $49.6 \pm 8.3$ & $51.1 \pm 6.8$ & $54.9 \pm 7.8$ & $53.0 \pm 7.7$ & $<0.001$ \\
\hline Male (\%) & 49.7 & 70.5 & 73.0 & 81.6 & $<0.001$ \\
\hline BMI $\left(\mathrm{kg} / \mathrm{m}^{2}\right)$ & $22.5 \pm 2.2$ & $26.5 \pm 2.2$ & $25.4 \pm 2.0$ & $29.6 \pm 3.1$ & $<0.001$ \\
\hline Waist circumference $(\mathrm{cm})$ & $78.9 \pm 6.5$ & $92.9 \pm 4.6$ & $84.2 \pm 4.2$ & $98.9 \pm 7.4$ & $<0.001$ \\
\hline $\operatorname{ASM}(\mathrm{kg})$ & $19.2 \pm 4.1$ & $22.9 \pm 4.4$ & $17.1 \pm 3.4$ & $21.9 \pm 4.4$ & $<0.001$ \\
\hline ASM/weight (\%) & $31 \pm 3$ & $30 \pm 3$ & $27 \pm 3$ & $27 \pm 3$ & $<0.001$ \\
\hline $\mathrm{ASM} / \mathrm{BMI}\left(\mathrm{m}^{2}\right)$ & $1.41 \pm 0.57$ & $1.13 \pm 0.32$ & $0.82 \pm 0.19$ & $0.77 \pm 0.16$ & $<0.001$ \\
\hline Body fat percentage $(\%)$ & $24.4 \pm 6.1$ & $28.3 \pm 5.8$ & $33.6 \pm 5.2$ & $35.7 \pm 5.2$ & $<0.001$ \\
\hline Current smoking (\%) & 19.7 & 27.5 & 18.5 & 29.0 & $<0.001$ \\
\hline Modest alcohol intake (\%) & 12.7 & 21.6 & 14.6 & 24.1 & $<0.001$ \\
\hline Regular exercise $(\%)$ & 29.9 & 25.6 & 26.9 & 22.7 & $<0.001$ \\
\hline $\mathrm{SBP}(\mathrm{mmHg})$ & $116(106-127)$ & $123(113-134)$ & $126(113-137)$ & $127(117-139)$ & $<0.001$ \\
\hline Triglycerides (mg/dl) & $89(65-128)$ & $117(85-162)$ & $115(84-160)$ & $134(99-191)$ & $<0.001$ \\
\hline HDL-C (mg/dl) & $55(46-66)$ & $50(44-61)$ & $47(41-56)$ & $46(39-55)$ & $<0.001$ \\
\hline Fasting glucose (mg/dl) & $90(84-97)$ & $94(88-103)$ & $94(87-104)$ & $97(90-109)$ & $<0.001$ \\
\hline hsCRP (mg/dl) & $0.04(0.03-0.08)$ & $0.07(0.04-0.15)$ & $0.08(0.04-0.16)$ & $0.12(0.06-0.24)$ & $<0.001$ \\
\hline
\end{tabular}

Values are expressed as means \pm standard deviation, percentages, or median (interquartile range).

BMI, body mass index; ASM, appendicular skeletal muscle mass; SBP, systolic blood pressure; HDL-C, high-density lipoprotein cholesterol.

performed subgroup analyses of factors affecting the risk of erosive esophagitis (Table 5). The pre-specified subgroup analysis did not show heterogeneity of the risk of erosive esophagitis from obesity and sarcopenia status, or significant interactions with age ( $<60$ vs. $\geq 60$ years), sex (women vs. men), smoking status (non-current vs. current smokers), alcohol intake (mild vs. modest), or regular exercise (no vs. yes).

\section{DISCUSSION}

In this large, cross-sectional study of asymptomatic men and women undergoing upper gastrointestinal tract endoscopies, we found that sarcopenic obese individuals were at higher risk of erosive esophagitis than obese individuals without sarcopenia. Our results also showed that non-obese sarcopenic individuals are at an increased risk of erosive esophagitis compared to obese individuals without sarcopenia. These associations persisted after adjusting for potential confounders and metabolic risk factors, and remained evident across all evaluated subgroups. Thus, our findings indicate that sarcopenia, regardless of the presence of central obesity, is associated with a greater risk of erosive esophagitis than is central obesity in the absence of sarcopenia.

To our knowledge, this is the first large scale study investigating obesity/sarcopenia and the risk of erosive esophagitis. Sarcopenia has become an important concept for understanding the impact of aging on health outcomes.
This condition is not only a physiological symptom of aging, but there is growing evidence that low muscle mass is a condition associated with a number of disorders [11-17]. Sarcopenia is common in elderly populations and contributes to functional limitations, disabilities, and frailty [18, 19]. Furthermore, several previous studies have found an association with increased risk for several disorders, such as cardiovascular disease, metabolic syndrome, and morbidity or mortality, in individuals with sarcopenia [11-17].

Previous evidence has shown a significant association between RE and obesity, especially central obesity [20-22]. Although the precise mechanisms that link central obesity and RE are not yet fully elucidated, multiple mechanisms have been implicated to account for this association. Central obesity, which is typically measured in terms of waist circumference, waist-hip ratio, or visceral adiposity, seems to be a more important predictor of RE than is general obesity [23]. This may be due to the mechanical effect of the increased pressure gradient caused by visceral adiposity inducing RE in individuals with central obesity $[8,24]$. The important changes in body composition associated with RE, including a decline in skeletal muscle and an increase in body fat, especially abdominal visceral fat, are noticed in individuals with sarcopenia [14, 25-27]. In our study, participants with sarcopenia had higher body fat percentages than did participants without sarcopenia. Thus, increased body fat, especially abdominal adiposity, 
Table 2: The risk of erosive esophagitis according to sarcopenic obesity groups

\begin{tabular}{lccccc}
\hline & \multicolumn{4}{c}{ Sarcopenic obesity groups } & \\
\cline { 2 - 5 } & Normal OR & $\begin{array}{c}\text { Obese OR (95\% } \\
\text { CI) }\end{array}$ & $\begin{array}{c}\text { Sarcopenic OR } \\
\mathbf{( 9 5 \% ~ C I ) ~}\end{array}$ & $\begin{array}{c}\text { Sarcopenic obese } \\
\text { OR (95\% CI) }\end{array}$ & $\boldsymbol{P}$ value \\
\hline Crude Model & 1.00 (reference) & $1.95(1.79-2.12)$ & $2.80(1.92-4.09)$ & $2.72(2.29-3.24)$ & $<0.001$ \\
Model 1 & 1.00 (reference) & $1.53(1.40-1.67)$ & $2.23(1.51-3.29)$ & $1.93(1.61-2.30)$ & $<0.001$ \\
Model 2 & 1.00 (reference) & $1.46(1.12-1.33)$ & $2.33(1.57-3.46)$ & $1.82(1.51-2.18)$ & $<0.001$ \\
Model 3 & 1.00 (reference) & $1.38(1.26-1.52)$ & $2.20(1.48-3.29)$ & $1.68(1.39-2.03)$ & $<0.001$ \\
\hline
\end{tabular}

Model 1 adjusted for age and sex, Model. 2 adjusted for age, sex, smoking status, alcohol intake, and regular exercise. Model. 3 adjusted for systolic blood pressure, triglycerides, HDL-cholesterol, and fasting glucose in addition to the factors included in model, 2.OR, odds ratio; CI, confidence intervals.

might contribute to RE through a mechanical effect in sarcopenic patients. In addition, skeletal muscle is a primary tissue for insulin-mediated glucose disposal; therefore, the low skeletal muscle mass in patients with sarcopenia reduces insulin-mediated glucose uptake and, subsequently, induces insulin resistance [28, 29]. Moreover, a recent study, using data from the National Health and Nutrition Examination Survey III, showed that sarcopenia exacerbates obesity-associated insulin resistance and dysglycemia [30]. Additionally, the sarcopenia-associated loss of skeletal muscle mass and gain of visceral adiposity were positively associated with blood pressure, triglyceride levels, hyperglycemia, and a number of other metabolic syndrome components [14, 28, 29]. Strong evidence also supports the association of metabolic syndrome with RE [31,32]. Our study also revealed that metabolic components, such as
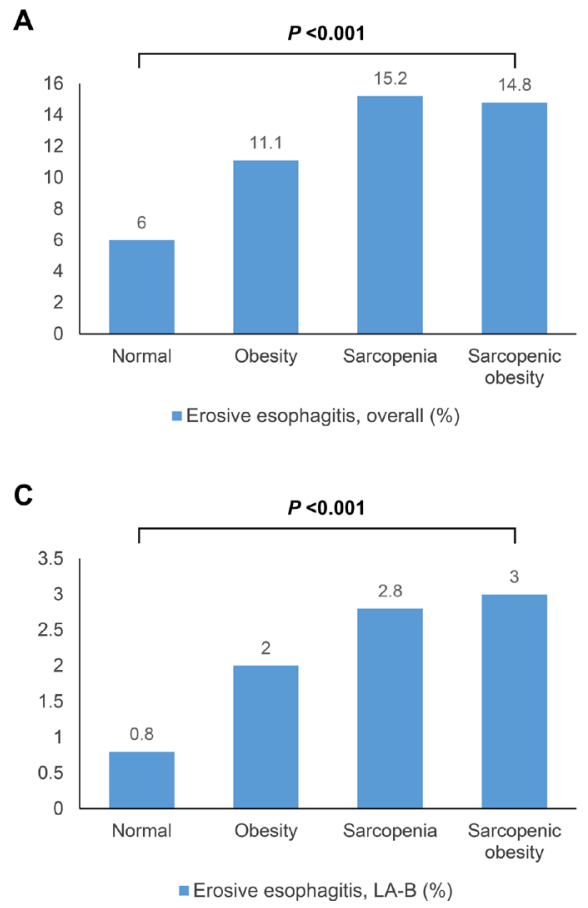

systolic blood pressure, fasting blood glucose levels, and triglyceride levels, were independent risk factors for erosive esophagitis. Further, individuals with sarcopenia had higher systolic blood pressures, and higher fasting blood glucose and triglyceride levels, than did those without sarcopenia.

Sarcopenia-induced chronic inflammation is another potential RE mechanism. Recently, esophageal inflammation involving a cytokine-mediated pathway, rather than reflux, has been proposed as a mechanism underlying the pathogenesis of RE [33]. Further, agerelated, chronic, low-grade inflammation is recognized as an important causative factor for sarcopenia [34], and cross-sectional and longitudinal studies support the association between inflammation and sarcopenia [34]. Adipocytokines, such as tumor necrosis factor- $\alpha$ and interleukin-6, are mainly involved in sarcopenia-
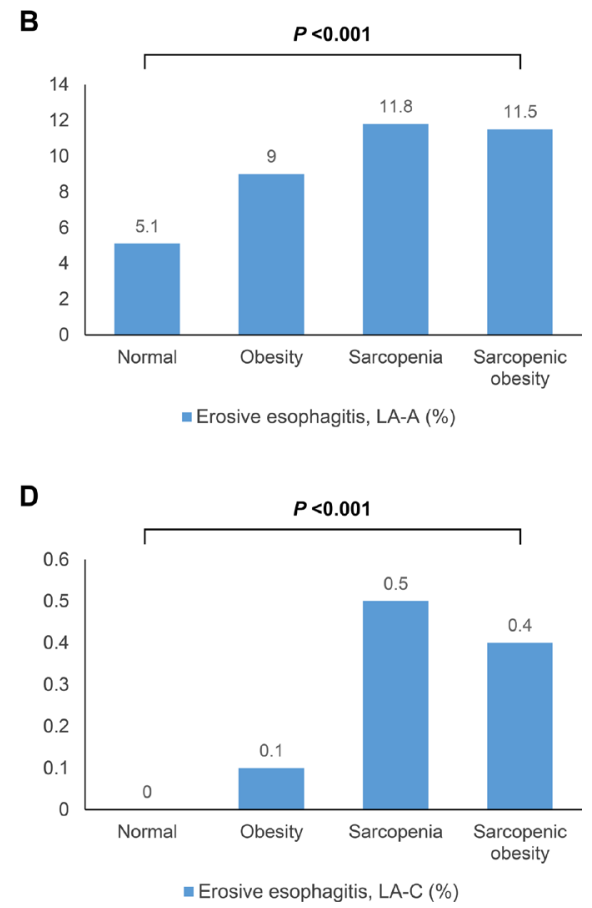

Figure 1: The prevalence of erosive esophagitis by Los Angeles classification according to sarcopenia and obesity status. 
Table 3: The risk of erosive esophagitis for sarcopenic and sarcopenic obese group comparing to obese group

\begin{tabular}{|c|c|c|c|c|c|c|}
\hline & \multicolumn{2}{|c|}{ Model 1} & \multicolumn{2}{|c|}{ Model 2} & \multicolumn{2}{|c|}{ Model 3} \\
\hline & OR (95\% CI) & $P$ value & OR $(95 \%$ CI) & $P$ value & OR $(95 \%$ CI $)$ & $P$ value \\
\hline Obese & 1.00 (reference) & & 1.00 (reference) & & 1.00 (reference) & \\
\hline Sarcopenic & $1.46(0.99-2.15)$ & 0.05 & $1.59(1.07-2.37)$ & 0.02 & $1.59(1.06-2.38)$ & 0.02 \\
\hline Sarcopenic obese & $1.26(1.05-1.51)$ & 0.01 & $1.24(1.03-1.50)$ & 0.03 & $1.22(1.01-1.47)$ & 0.04 \\
\hline
\end{tabular}

Model 1 adjusted for age and sex. Model 2 adjusted for age, sex, smoking status, alcohol intake, and regular exercise. Model 3 adjusted for systolic blood pressure, triglycerides, HDL-cholesterol, and fasting glucose in addition to the factors included in model 2. OR, odds ratio; CI, confidence intervals.

associated inflammation [34]. However, further research is needed to understand the underlying mechanisms of insulin resistance, inflammation, and metabolic syndrome associated with sarcopenia and their roles in RE.

Several limitations need to be a considered when interpreting the results of our study. First, the crosssectional design of this study makes it impossible to determine any causality for the association. Second, although BIA is a convenient and safe method for assessing skeletal muscle mass index, it is not the gold standard method; the gold standards remain dual-energy X-ray absorptiometry, computed tomography, and magnetic resonance. However, several studies have validated the use of BIA, compared with either absorptiometry or magnetic resonance; these studies reported BIA to provide an accurate estimate of skeletal muscle mass index [3538]. Another limitation of BIA is that it cannot provide information on muscle quality, such as gait speed or grip strength; such characterizations describe sarcopenia in a functional way, not solely on the basis of muscle mass. Third, inter-observer variations in the endoscopic diagnoses of erosive esophagitis were not evaluated. However, experienced board-certified gastroenterologists performed the endoscopies, and erosive esophagitis was clearly defined; the same classification system was used for all of the participants. Finally, this study focused on healthy participants who underwent routine health check-ups; thus, our findings may not generalize to other populations.

This study also has several strengths. First, its large sample size and the adjustment for potential confounding factors of erosive esophagitis allowed us to minimize temporal bias. Additional strengths include the use of high-quality, standardized anthropometric measurements; the incorporation of an epidemiological questionnaire regarding lifestyle factors, and the inclusion of various laboratory studies.

In conclusion, this study showed that sarcopenia is associated with an increased risk of erosive esophagitis, regardless of the presence of central obesity. Furthermore, sarcopenia without central obesity and sarcopenic obesity also demonstrated increased risk of erosive esophagitis, compared with obesity in the absence of sarcopenia. The risk of erosive esophagitis appears to have been mediated by metabolic risk factors. Further longitudinal studies are needed to confirm our findings and to elucidate the causal relationship between sarcopenia and erosive esophagitis.

\section{MATERIALS AND METHODS}

\section{Study population}

We conducted a retrospective, cross-sectional study of healthy men and women, who underwent a routine health check-up that included endoscopy. The participants underwent their health check-up at the Center for Health Promotion, Samsung Medical Center, Seoul, South Korea, between August 2006 and December 2011. We screened consecutive participants who underwent esophagogastroduodenoscopy (EGD) during the study period. Since our objective was to evaluate the association between obesity/sarcopenic status and RE, we included participants who underwent both EGD and anthropometric measurements, including waist circumference, body weight, and appendicular skeletal muscle mass (ASM) $(n=37,815)$. We excluded participants meeting any of the following exclusion criteria: gastric cancer $(n=124)$, esophageal cancer $(n=25)$, history of other malignancy ( $n=772)$, Barrett's esophagitis $(n=37)$, or previous gastric surgery $(n=322)$. We also excluded participants missing data on important covariates: incomplete endoscopic report $(n=649)$, alcohol consumption $(n=$ $1,164)$, smoking history $(n=1,487)$, exercise $(n=1,083)$, lipid profiles $(n=327)$, fasting blood glucose levels $(n$ $=1)$, or systolic blood pressure $(n=7)$. Finally, 32,762 asymptomatic participants who underwent screening EGD and anthropometric measurements were included in this study (Supplementary Figure 1). This study was approved by the Samsung Medical Center Institutional Review Board and was conducted in accordance with the Declaration of Helsinki and current legal regulations in Korea. Institutional Review Board approval was obtained, but did not require specific informed consent because the study used only de-identified data collected for clinical purposes as part of the health screening. However, informed consent was obtained from all subjects for their examinations during the health check-up. 


\section{Table 4: The risk of erosive esophagitis by LA classification according to sarcopenia status}

\begin{tabular}{lccc}
\hline & \multicolumn{3}{c}{ Multivariable-adjusted OR (95\% CI) } \\
\cline { 2 - 4 } & Z LA-A & $\geq$ LA-B & $\geq$ LA-C \\
\hline Cut-off values for sarcopenia & & & \\
Normal & 1.00 (reference) & 1.00 (reference) & 1.00 (reference) \\
Class I sarcopenia $^{\mathrm{a}}$ & $1.19(1.08-1.32)$ & $1.59(1.29-1.96)$ & $2.60(0.97-6.99)$ \\
Class II sarcopenia $^{\mathrm{b}}$ & $1.51(1.26-1.81)$ & $1.74(1.42-2.13)$ & $4.93(1.24-19.57)$ \\
$P$ for trend & $<0.001$ & 0.004 & 0.046 \\
Per 1\% decrease in ASM/weight & $1.04(1.01-1.06)$ & $1.07(1.01-1.13)$ & $1.30(1.10-1.55)$ \\
\hline
\end{tabular}

Multivariable model was adjusted for age, sex, waist circumference, smoking status, alcohol intake, and regular exercise. aparticipants with class I sarcopenia were those with weight-adjusted skeletal muscle mass index between one and two standard deviations below the sex-specific values measured in young healthy adults.

${ }^{b}$ Participants with class II sarcopenia were those with weight-adjusted skeletal muscle mass index more than two standard deviations below the sex-specific values measured in young healthy adults. LA, Los Angeles; OR, odds ratio; CI, confidence intervals; ASM, appendicular skeletal muscle mass.

\section{Data collection}

The comprehensive health-screening program included anthropometric measurements, endoscopy, serum biochemical measurements, and completion of an epidemiological questionnaire regarding smoking habits, alcohol consumption, physical activity, personal medical history, and family history of cancer [39]. Smoking status was categorized as never, former, or current smoker. Alcohol consumption status was categorized as either mild ( $\leq 10 \mathrm{~g} /$ day) or modest (>10 g/day). Regular exercise was defined as exercising $\geq 3$ times/week at a moderate intensity. The participants' weights and heights were measured while wearing light clothing and bare feet. Weight and height were determined to the nearest $0.1 \mathrm{~kg}$ and $0.1 \mathrm{~cm}$, respectively; the body mass index (BMI) was calculated as weight $(\mathrm{kg})$ divided by height $(\mathrm{m})$ squared $\left(\mathrm{kg} / \mathrm{m}^{2}\right)$. Waist circumference was measured in a horizontal plane at the midpoint between the inferior margin of the last rib and the superior iliac crest. Blood pressure was measured, using an automated blood pressure monitor (Dinamap PRO 100; GE Healthcare, Milwaukee, WI), while the participant was in the seated position after $>5$ minutes of quiet rest.

After $\mathrm{a} \geq 12$-h fast, blood samples were collected in the morning and analyzed in the hospital's clinical laboratory. Serum glucose levels were measured using the hexokinase/ glucose-6-phosphate dehydrogenase method and an autoanalyzer (7600 Modular Dp-110, Hitachi, Tokyo, Japan). Serum levels of glucose, total cholesterol, triglycerides, and high-density lipoprotein-cholesterol (HDL-C) were measured using enzymatic colorimetric and liquid-selective detergent methods in the same type of autoanalyzer.

\section{Definitions of obesity and sarcopenia}

To estimate the ASM, bioelectrical impedance analysis (BIA) was performed using an Inbody 720 (Biospace, Seoul, Korea), which is a convenient and cost-effective tool for assessing impedance in various body segments, including the four limbs and trunk [38]. ASM was calculated as the sum of the lean muscle mass of the bilateral upper and lower limbs. The skeletal muscle mass index, a validated measure of sarcopenia, was calculated as: the skeletal muscle mass index $(\%)=$ ASM (kg)/body weight $(\mathrm{kg}){ }^{*} 100$ [36]. Sarcopenia was defined as a skeletal muscle mass index more than two standard deviations below the sex-specific values measured in young healthy adults, according to national health examinations in Korea (skeletal muscle mass index $<29.1$ in males and $<23.0$ in females) [40].

Obesity was defined as central obesity (waist circumference $\geq 90 \mathrm{~cm}$ in men and $\geq 85 \mathrm{~cm}$ in women) according to the Korean Society for the Study of Obesity [41]. Study participants were classified into four groups based on sarcopenia and obesity status: non-sarcopenic and non-obese (normal) group; sarcopenic and non-obese (sarcopenic) group; non-sarcopenic and obese (obese) group; and sarcopenic and obese (sarcopenic obese) group.

\section{Esophagogastroduodenoscopy}

Thirty-four experienced board-certified gastroenterologists performed each endoscopy using a gastroscope (Olympus GIF-Q260; Olympus Medical Systems, Tokyo, Japan). All endoscopists completed a gastroenterology fellowship. Median year of graduation from medical school of the 34 gastroenterologists is 1997 (range, 1989-2003). The primary endpoint was the presence of erosive esophagitis noted during EGD. Erosive esophagitis was defined as the presence of definite mucosal breaks (erosions) and was classified according to the Los Angeles classification system [42].

\section{Statistical analysis}

Continuous variables are reported as means \pm standard deviations, while categorical variables are 
Table 5: The risk of erosive esophagitis by sarcopenic obesity groups in clinically-relevant subgroups

\begin{tabular}{|c|c|c|c|c|c|}
\hline \multirow[b]{2}{*}{ Subgroup } & \multicolumn{4}{|c|}{ Sarcopenic obesity groups } & \multirow[b]{2}{*}{$\mathbf{P}$ for interaction } \\
\hline & Normal & Obese & Sarcopenic & $\begin{array}{c}\text { Sarcopenic } \\
\text { obese }\end{array}$ & \\
\hline Age & & & & & 0.25 \\
\hline$<60$ years $(n=27,957)$ & reference & $1.52(1.33-1.82)$ & $2.77(1.80-4.27)$ & $1.91(1.46-2.55)$ & \\
\hline$\geq 60$ years $(n=4,805)$ & reference & $1.33(1.14-1.72)$ & $2.03(1.10-4.55)$ & $1.48(1.17-2.16)$ & \\
\hline Sex & & & & & 0.69 \\
\hline Women $(n=14,373)$ & reference & $1.45(1.10-1.91)$ & $1.73(1.18-6.52)$ & $2.45(1.27-4.74)$ & \\
\hline Men $(n=18,389)$ & reference & $1.46(1.32-1.60)$ & $2.32(1.54-3.51)$ & $1.74(1.44-2.11)$ & \\
\hline Current Smoking & & & & & 0.5 \\
\hline No $(n=25,584)$ & reference & $1.43(1.27-1.61)$ & $2.11(1.32-3.38)$ & $1.94(1.54-2.44)$ & \\
\hline Yes $(n=7,178)$ & reference & $1.51(1.31-1.74)$ & $2.65(1.28-5.51)$ & $1.56(1.15-2.12)$ & \\
\hline Alcohol intake & & & & & 0.96 \\
\hline Mild $(n=27,736)$ & reference & $1.47(1.31-1.63)$ & $2.20(1.41-3.44)$ & $1.83(1.47-2.28)$ & \\
\hline Modest $(n=5,026)$ & reference & $1.46(1.23-1.74)$ & $2.61(1.10-6.16)$ & $1.70(1.21-2.39)$ & \\
\hline Regular exercise & & & & & 0.06 \\
\hline No $(23,395)$ & reference & $1.47(1.32-1.64)$ & $2.84(1.84-4.38)$ & $1.96(1.60-2.41)$ & \\
\hline Yes $(9,367)$ & reference & $1.44(1.21-1.71)$ & $2.14(1.21-4.72)$ & $1.44(1.13-1.92)$ & \\
\hline
\end{tabular}

Estimated from logistic regression models adjusted for age, sex, smoking status, alcohol intake, regular exercise, and metabolic variables

presented as percentages. Continuous variables were compared between groups using one-way ANOVA; categorical variables were compared using the Chi-squared test. Logistic regression models were used to estimate odds ratios (ORs) and 95\% confidence intervals (CIs) for erosive esophagitis in the sarcopenic obese, sarcopenic, and obese groups using the normal group as the reference group. To evaluate whether sarcopenia imparts a greater risk for the development of erosive esophagitis than obesity, we estimated adjusted ORs in the sarcopenic obese and sarcopenic groups, using the obese group as the reference group. We used three models, with increasing levels of adjustment, to account for potential confounders. Model 1 was adjusted for age (/year) and sex. Model 2 was further adjusted for smoking status (never/past vs. current smoker), alcoholic intake (mild vs. modest), and regular exercise (yes vs. no). Model 3 was further adjusted for metabolic variables, including systolic blood pressure, triglyceride level, HDL-C level, and fasting glucose level, to account for the possible mediation of the association between sarcopenia and erosive esophagitis by these metabolic risk factors.

We conducted two additional types of doseresponse analyses. First, we conducted the multivariable analysis based on the severity categories of sarcopenia, and estimated adjusted ORs with 95\% CIs for erosive esophagitis comparing categories with the normal category. We categorized the sarcopenia into 3 groups according to severity: normal, class I sarcopenia, and class II sarcopenia. Subjects with class I sarcopenia were those with weight-adjusted skeletal muscle mass index between one and two standard deviations below the sexspecific values measured in young healthy adults. Subjects with class II sarcopenia were those with weight-adjusted skeletal muscle mass index more than two standard deviations below the sex-specific values measured in young healthy adults. To evaluate the linear trends of risk, we used a continuous variable with the category number and tested its statistical significance in the regression models. Second, we estimated the adjusted ORs with 95\% CIs associated with weight-adjusted skeletal muscle mass index decrease of $1 \%$ using ASM/weight as a continuous variable in the logistic regression model.

We conducted subgroup analyses to identify interactions between obesity/sarcopenic status and clinically relevant groups, defined by age $(<60$ years vs. $\geq 60$ years), sex (women vs. men), smoking status (non-current vs. current smokers), alcohol intake (mild vs. modest), and regular exercise (no vs. yes). Subgroup interactions were tested using likelihood ratio tests and comparing models with and without multiplicative interaction terms. Statistical analyses were performed using SAS version 9.4 (SAS Institute, Cary, NC); a $P$-value $<0.05$ was considered statistically significant.

\section{Abbreviations}

$$
\text { RE, Reflux esophagitis; EGD, }
$$
esophagogastroduodenoscopy; OR, odds ratios; CI, confidence interval; HDL-C, high-density lipoprotein- 
cholesterol; BIA, Bioelectrical impedance analysis; ASM, appendicular skeletal muscle mass.

\section{ACKNOWLEDGMENTS}

None.

\section{CONFLICTS OF INTEREST}

The authors declare no any conflicts of interest in this work.

\section{FUNDING}

None.

\section{REFERENCES}

1. Peery AF, Dellon ES, Lund J, Crockett SD, McGowan CE, Bulsiewicz WJ, Gangarosa LM, Thiny MT, Stizenberg K, Morgan DR, Ringel Y, Kim HP, Dibonaventura MD, et al. Burden of gastrointestinal disease in the United States: 2012 update. Gastroenterology. 2012; 143:1179-87 e1-3. https:// doi.org/10.1053/j.gastro.2012.08.002.

2. El-Serag HB, Sweet S, Winchester CC, Dent J. Update on the epidemiology of gastro-oesophageal reflux disease: a systematic review. Gut. 2014; 63:871-80. https://doi. org/10.1136/gutjnl-2012-304269.

3. Lagergren J. Influence of obesity on the risk of esophageal disorders. Nat Rev Gastroenterol Hepatol. 2011; 8:340-7. https://doi.org/10.1038/nrgastro.2011.73.

4. Turati F, Tramacere I, La Vecchia C, Negri E. A metaanalysis of body mass index and esophageal and gastric cardia adenocarcinoma. Ann Oncol. 2013; 24:609-17. https://doi.org/10.1093/annonc/mds244.

5. Edelstein ZR, Farrow DC, Bronner MP, Rosen SN, Vaughan TL. Central adiposity and risk of Barrett's esophagus. Gastroenterology. 2007; 133:403-11. https:// doi.org/10.1053/j.gastro.2007.05.026.

6. Rubenstein JH, Kao JY, Madanick RD, Zhang M, Wang M, Spacek MB, Donovan JL, Bright SD, Shaheen NJ. Association of adiponectin multimers with Barrett's oesophagus. Gut. 2009; 58:1583-9. https://doi.org/10.1136/gut.2008.171553.

7. Anggiansah R, Sweis R, Anggiansah A, Wong T, Cooper D, Fox M. The effects of obesity on oesophageal function, acid exposure and the symptoms of gastro-oesophageal reflux disease. Aliment Pharmacol Ther. 2013; 37:555-63. https:// doi.org/10.1111/apt.12208.

8. Robertson EV, Derakhshan MH, Wirz AA, Lee YY, Seenan JP, Ballantyne SA, Hanvey SL, Kelman AW, Going JJ, McColl KE. Central obesity in asymptomatic volunteers is associated with increased intrasphincteric acid reflux and lengthening of the cardiac mucosa. Gastroenterology. 2013; 145:730-9. https://doi.org/10.1053/j.gastro.2013.06.038.
9. Souza RF, Huo X, Mittal V, Schuler CM, Carmack SW, Zhang HY, Zhang X, Yu C, Hormi-Carver K, Genta RM, Spechler SJ. Gastroesophageal reflux might cause esophagitis through a cytokine-mediated mechanism rather than caustic acid injury. Gastroenterology. 2009; 137:177684. https://doi.org/10.1053/j.gastro.2009.07.055.

10. Gomes MJ, Martinez PF, Pagan LU, Damatto RL, Cezar MDM, Lima ARR, Okoshi K, Okoshi MP. Skeletal muscle aging: influence of oxidative stress and physical exercise. Oncotarget. 2017; 8:20428-40. https://doi.org/10.18632/ oncotarget.14670.

11. Beaudart C, Zaaria M, Pasleau F, Reginster JY, Bruyere O. Health Outcomes of Sarcopenia: A Systematic Review and Meta-Analysis. PLoS One. 2017; 12:e169548. https://doi. org/10.1371/journal.pone.0169548.

12. Chin SO, Rhee SY, Chon S, Hwang YC, Jeong IK, Oh S, Ahn KJ, Chung HY, Woo JT, Kim SW, Kim JW, Kim YS, Ahn HY. Sarcopenia is independently associated with cardiovascular disease in older Korean adults: the Korea National Health and Nutrition Examination Survey (KNHANES) from 2009. PLoS One. 2013; 8:e60119. https://doi.org/10.1371/journal.pone.0060119.

13. $\mathrm{Hu} \mathrm{YB}, \mathrm{Chen} \mathrm{Z}, \mathrm{Fu} \mathrm{RQ}$. The association between sarcopenia and non-alcoholic fatty liver disease. J Hepatol. 2017; 66:243-4. https://doi.org/10.1016/j.jhep.2016.09.023.

14. Kim TN, Choi KM. The implications of sarcopenia and sarcopenic obesity on cardiometabolic disease. J Cell Biochem. 2015; 116:1171-8. https://doi.org/10.1002/ jcb.25077.

15. Nardelli S, Lattanzi B, Torrisi S, Greco F, Farcomeni A, Gioia S, Merli M, Riggio O. Sarcopenia Is Risk Factor for Development of Hepatic Encephalopathy After Transjugular Intrahepatic Portosystemic Shunt Placement. Clin Gastroenterol Hepatol. 2016. https://doi.org/10.1016/j. cgh.2016.10.028.

16. Petta S, Ciminnisi S, Di Marco V, Cabibi D, Camma C, Licata A, Marchesini G, Craxi A. Sarcopenia is associated with severe liver fibrosis in patients with non-alcoholic fatty liver disease. Aliment Pharmacol Ther. 2017; 45:510-8. https://doi.org/10.1111/apt.13889.

17. Voron T, Tselikas L, Pietrasz D, Pigneur F, Laurent A, Compagnon P, Salloum C, Luciani A, Azoulay D. Sarcopenia Impacts on Short- and Long-term Results of Hepatectomy for Hepatocellular Carcinoma. Ann Surg. 2015; 261:1173-83. https://doi.org/10.1097/ SLA.0000000000000743.

18. Stenholm S, Harris TB, Rantanen $T$, Visser $M$, Kritchevsky SB, Ferrucci L. Sarcopenic obesity: definition, cause and consequences. Curr Opin Clin Nutr Metab Care. 2008; 11:693-700. https://doi.org/10.1097/ MCO.0b013e328312c37d.

19. Walston JD. Sarcopenia in older adults. Curr Opin Rheumatol. 2012; 24:623-7. https://doi.org/10.1097/ BOR.0b013e328358d59b. 
20. Corley DA, Kubo A. Body mass index and gastroesophageal reflux disease: a systematic review and meta-analysis. Am J Gastroenterol. 2006; 101:2619-28. https://doi.org/10.1111/ j.1572-0241.2006.00849.x.

21. El-Serag H. The association between obesity and GERD: a review of the epidemiological evidence. Dig Dis Sci. 2008; 53:2307-12. https://doi.org/10.1007/s10620-008-0413-9.

22. Murray L, Johnston B, Lane A, Harvey I, Donovan J, Nair P, Harvey R. Relationship between body mass and gastrooesophageal reflux symptoms: The Bristol Helicobacter Project. Int J Epidemiol. 2003; 32:645-50. https://doi.org/

23. Singh S, Sharma AN, Murad MH, Buttar NS, El-Serag HB, Katzka DA, Iyer PG. Central adiposity is associated with increased risk of esophageal inflammation, metaplasia, and adenocarcinoma: a systematic review and meta-analysis. Clin Gastroenterol Hepatol. 2013; 11:1399-412 e7. https:// doi.org/10.1016/j.cgh.2013.05.009.

24. Lee YY, McColl KE. Disruption of the gastroesophageal junction by central obesity and waist belt: role of raised intra-abdominal pressure. Dis Esophagus. 2015; 28:318-25. https://doi.org/10.1111/dote.12202.

25. Baumgartner RN. Body composition in healthy aging. Ann N Y Acad Sci. 2000; 904:437-48.

26. Myint PK, Welch AA. Healthier ageing. BMJ. 2012; 344:e1214. https://doi.org/10.1136/bmj.e1214.

27. Zamboni M, Mazzali G, Fantin F, Rossi A, Di Francesco V. Sarcopenic obesity: a new category of obesity in the elderly. Nutr Metab Cardiovasc Dis. 2008; 18:388-95. https://doi. org/10.1016/j.numecd.2007.10.002.

28. Lee SW, Youm Y, Lee WJ, Choi W, Chu SH, Park YR, Kim HC. Appendicular skeletal muscle mass and insulin resistance in an elderly korean population: the korean social life, health and aging project-health examination cohort. Diabetes Metab J. 2015; 39:37-45. https://doi.org/10.4093/dmj.2015.39.1.37.

29. Lim S, Kim JH, Yoon JW, Kang SM, Choi SH, Park YJ, Kim KW, Lim JY, Park KS, Jang HC. Sarcopenic obesity: prevalence and association with metabolic syndrome in the Korean Longitudinal Study on Health and Aging (KLoSHA). Diabetes Care. 2010; 33: 1652-4. https://doi. org/10.2337/dc10-0107.

30. Srikanthan P, Hevener AL, Karlamangla AS. Sarcopenia exacerbates obesity-associated insulin resistance and dysglycemia: findings from the National Health and Nutrition Examination Survey III. PLoS One. 2010; 5: e10805. https://doi.org/10.1371/journal.pone.0010805.

31. Chung SJ, Kim D, Park MJ, Kim YS, Kim JS, Jung HC, Song IS. Metabolic syndrome and visceral obesity as risk factors for reflux oesophagitis: a cross-sectional casecontrol study of 7078 Koreans undergoing health checkups. Gut. 2008; 57: 1360-5. https://doi.org/10.1136/ gut.2007.147090.

32. Wu P, Ma L, Dai GX, Chen Y, Tong YL, Wang C, Yao LW, Jiang YX, Xu SC, Ai ZS. The association of metabolic syndrome with reflux esophagitis: a case-control study.
Neurogastroenterol Motil. 2011; 23:989-94. https://doi. org/10.1111/j.1365-2982.2011.01786.x.

33. Dunbar KB, Agoston AT, Odze RD, Huo X, Pham TH, Cipher DJ, Castell DO, Genta RM, Souza RF, Spechler SJ. Association of Acute Gastroesophageal Reflux Disease With Esophageal Histologic Changes. JAMA. 2016; 315:2104-12. https://doi.org/10.1001/jama.2016.5657.

34. Beyer I, Mets T, Bautmans I. Chronic low-grade inflammation and age-related sarcopenia. Curr Opin Clin Nutr Metab Care. 2012; 15:12-22. https://doi.org/10.1097/ MCO.0b013e32834dd297.

35. Bosaeus I, Wilcox G, Rothenberg E, Strauss BJ. Skeletal muscle mass in hospitalized elderly patients: comparison of measurements by single-frequency BIA and DXA. Clin Nutr. 2014; 33:426-31. https://doi.org/10.1016/j. clnu.2013.06.007.

36. Janssen I, Heymsfield SB, Ross R. Low relative skeletal muscle mass (sarcopenia) in older persons is associated with functional impairment and physical disability. J Am Geriatr Soc. 2002; 50:889-96.

37. Marini E, Buffa R, Saragat B, Coin A, Toffanello ED, Berton L, Manzato E, Sergi G. The potential of classic and specific bioelectrical impedance vector analysis for the assessment of sarcopenia and sarcopenic obesity. Clin Interv Aging. 2012; 7:585-91. https://doi.org/10.2147/CIA. S38488.

38. Rangel Peniche DB, Raya Giorguli G, Aleman-Mateo H. Accuracy of a predictive bioelectrical impedance analysis equation for estimating appendicular skeletal muscle mass in a non-Caucasian sample of older people. Arch Gerontol Geriatr. 2015; 61:39-43. https://doi.org/10.1016/j. archger.2015.03.007.

39. Kim TJ, Sinn DH, Min YW, Son HJ, Kim JJ, Chang Y, Baek SY, Ahn SH, Lee H, Ryu S. A cohort study on Helicobacter pylori infection associated with nonalcoholic fatty liver disease. J Gastroenterol. 2017. https://doi.org/10.1007/ s00535-017-1337-y.

40. Kim YS, Lee Y, Chung YS, Lee DJ, Joo NS, Hong D, Song G, Kim HJ, Choi YJ, Kim KM. Prevalence of sarcopenia and sarcopenic obesity in the Korean population based on the Fourth Korean National Health and Nutritional Examination Surveys. J Gerontol A Biol Sci Med Sci. 2012; 67:1107-13. https://doi.org/10.1093/gerona/gls071.

41. Lee SY, Park HS, Kim DJ, Han JH, Kim SM, Cho GJ, Kim DY, Kwon HS, Kim SR, Lee CB, Oh SJ, Park CY, Yoo HJ. Appropriate waist circumference cutoff points for central obesity in Korean adults. Diabetes Res Clin Pract. 2007; 75:72-80. https://doi.org/10.1016/j.diabres.2006.04.013.

42. Lundell LR, Dent J, Bennett JR, Blum AL, Armstrong D, Galmiche JP, Johnson F, Hongo M, Richter JE, Spechler SJ, Tytgat GN, Wallin L. Endoscopic assessment of oesophagitis: clinical and functional correlates and further validation of the Los Angeles classification. Gut. 1999; 45:172-80. 